\title{
Adhesion between graphene and polymers: A surface analysis perspective
}

\author{
M. C. F. da Costa, M. R. M. Sousa, D. R. G. Larrudé, G. J. M. Fechine* \\ Graphene and Nanomaterials Research Centre - MackGraphe, Mackenzie Presbyterian University, 01302-907 São Paulo, \\ Brazil
}

Received 13 June 2018; accepted in revised form 8 August 2018

\begin{abstract}
Efficient adhesion between polymers and two-dimensional materials, such as graphene, is fundamental and crucial for the development of flexible devices or special coating materials as well as defining the quality of the transfer processes for these materials. Here, contact angle (CA) measurements of four distinct polymers, low-density polyethylene - LDPE, polypropylene - PP, poly (butylene adipate-co-terephthalate) - PBAT and poly (vinylidene-fluoride-co-trifluoroethylene) PVDF-TrFE, and graphene achieved by chemical vapor deposition (CVD) were used to understand the adhesion phenomena between such materials. The CA measurements were carried out at specific thermal conditions mimicking a transfer process that is based on direct contact of CVD graphene and polymers above their melting temperature (Direct Dry Transfer - DDT). Surface analysis allowed the efficiency of such transfer method to be pre-estimated owing to an understanding of the adhesion properties of both materials by comparing their polar and dispersive components values. However, rheological properties and chemical structures seemed to be equally important in this evaluation, either by molecular weight modification or introduction of chemical groups onto the surface of polymer films. The results allowed for an understanding of the role of the main factors in adhesion phenomena between graphene and polymers and how they can be used to improve graphene coating during transfer processes.
\end{abstract}

Keywords: nanomaterials, coatings, adhesion, polymers, graphene

\section{Introduction}

The advent of graphene has opened new avenues of research for a variety of applications, especially for flexible devices and coating materials with different functionalities by using polymers as support substrates $[1,2]$. This material offers properties that are not conventional to others traditionally used, for example, a well-established thermal, electrical, optical, and mechanical behavior. From a surface point of view, the properties of graphene make this material very attractive for several applications, from the tribological area for wear protection [3] to surface coatings for flexible devices [4]. Besides, thanks to its impermeability to liquids and gases, graphene can be used as protection against degradation processes [5].
In order to assemble such structures, the understanding of surface phenomenon and interface between polymers and two-dimensional (2D) materials is essential. Studying the effects involving the control of interfaces, in this case, is both fundamental and crucial to provide efficient adherence between materials $[6,7]$, which is key for devices or special coatings with suitable design and performance from an application perspective.

The graphene produced via chemical vapor deposition (CVD), a scalable method for synthesizing materials in a large-area and of high quality, is frequently deposited onto the surface of metallic foils, such as copper $(\mathrm{Cu})$ and nickel $(\mathrm{Ni})$, due to their carbon solubility [8]. Therefore, the graphene film must be 
transferred from its original growth substrate to a given target surface, to not only prepare flexible devices, but to also modify the surface properties of polymers. The final structure with singular physical and chemical properties makes a vast number of applications such as thin surface coatings feasible in several fields from biomedicine (acting as implants or biosensors), electronics, photonics, optoelectronics, and organic electrodes, such as in touch-screen technology or light-emitting diodes (LEDs) [8, 9]. The Direct Dry Transfer (DDT) [10] is a technique used for this accomplishment by means of direct contact between the molten polymer and graphene that is transferred using only moderate heat and pressure and the later mechanical peeling of the original graphene substrate. Hence, adhesion between polymer films and CVD graphene deposited onto solid surfaces plays a vital role to guarantee a well-succeeded transfer result. This process occurs at high temperatures, in which the molten polymer with viscoelastic behavior can be in direct contact with the graphene $\mathrm{Cu}$ foil $[10,11]$. The results presented in these manuscripts indicated that the rheological behavior of polymers in such transfer temperatures is of paramount importance to achieve satisfactory results and a good quality graphene response since it directly intermeddle in the surface adhesion between CVD graphene and polymers.

The surface free energy (SFE) calculation is a tool frequently used to define charge interactions that occur on the surfaces and estimate the adhesion capacity of different materials [12]. There are some complex models to describe these interactions on wettability and adhesion [13, 14], but a simplified one involving polar $\left(\gamma_{\mathrm{p}}\right)$ and dispersive $\left(\gamma_{\mathrm{d}}\right)$ components can be exploited to explain permanent and induced dipoles effects, such as hydrogen bonds or even secondary interactions including charge distribution of substances, such as van der Waals bonds, respectively $[15,16]$. This model, based on Young's Equation, divides the SFE of solids into polar and dispersive components and it is usually obtained using models considering contact angle $(C A)$ measurements provided by using different test liquids. In this way, surface adhesions may be inferred by the comparison of these components for different materials. It means that materials with higher adhesion will present component values more similar to each other (polar-polar and dispersive-dispersive components) $[15,17,18]$.
Specifically, topics involving graphene-water interactions and its wettability have been discussed in numerous papers; however, it remains elusive and contradictory in literature $[1,2,4,19-22]$. Several authors have studied such surface properties and none of them considered the effect of it on the adhesion between graphene and polymers. The achievement of good adhesion leads to a better performance of the transfer process providing a high-quality of transferred graphene $[10,11,22]$. Usually, for polymers, contact angle measurements are made at room temperature $[15,23]$, but for DDT technique [10], in which temperature has a strong contribution, they may be not valid for this purpose since heat effects can interfere in the contact angle values achieved. Hence, for the first time, static $C A$ measurements were completed at high temperatures for polymers (low-density polyethylene - LDPE, polypropylene - PP, poly (butylene adipate-co-terephthalate) - PBAT and poly (vinylidene-fluoride-co-trifluoroethylene) - PVDFTrFE) in order to mimic thermal conditions that occur during transfer processes. Such measurements allowed the adhesion to be inferred and make possible the qualification of polymers suitability for graphene transfer. In addition, rheological properties were evaluated for the purpose of obtaining data about adhesion by using the Dahlquist Criterion and complex viscosity analysis $[10,23]$. Furthermore, the influence of molecular weight modification of polymers and chemical structure variation were analyzed to better understand the adhesion between polymers/ graphene using rheological tests and Fourier-transform infrared spectroscopy (FTIR), respectively.

\section{Experimental section}

\subsection{Materials}

Low-density polyethylene (LDPE) and polypropylene (PP), both from Braskem (Brazil), poly (butylene adipate-co-terephthalate) (PBAT) from BASF, and poly (vinylidene-fluoride-co-trifluoroethylene) (PVDF-TrFE) from Solvay (Belgium) were selected in order to evaluate differentiated polymers for the wide range of possible graphene-polymer applications. In addition to that, they present differing chemical structural and SFE values.

\subsection{CVD graphene growth conditions}

Monolayer graphene films were produced through chemical vapor deposition technique using a $25 \mu \mathrm{m}$ copper $(\mathrm{Cu})$ foil $(99.98 \%$ purity) as the catalyst 
substrate within a quartz tube using a hot wall furnace and low-pressure homemade CVD system. Initially, the copper foil was annealed for 40 minutes with a controlled hydrogen flow to stabilize the metallic surface by removing the copper oxide layer naturally formed and increasing its grain size according to morphology rearrangement and removal of structural defects. Afterwards, the growth process was achieved by the insertion of methane as a carbon source in addition to the hydrogen flow at an established range for 40 minutes at $1000^{\circ} \mathrm{C}$ to grow single-layer graphene sheets on the metallic surface [24].

\subsection{Polymer films fabrication}

Polymer pellets (LDPE, PP, PBAT) and powder (PVDF-TrFE) were transformed into films by using an uniaxial hydraulic press machine (UHPM) SOLAB SL 12/20 with tunable parameters for each polymer. Patterned pressure $\left(35,36 \mathrm{kgf} / \mathrm{cm}^{2}\right)$, pressure time (2 minutes), and temperature value above the melting point of polymers were applied to the set-up in order to produce films of 100-300 $\mu$ m thick. The polymer films were characterized using FourierTransform Infrared Spectroscopy (FTIR) to evaluate their chemical structure stability. They were also used to perform contact angle measurements at high temperatures with the view to mimicking thermal conditions during transfer processes.

\subsection{Contact angle measurements and Surface Free Energy calculation}

Taking into account that the adhesion process takes place at high temperatures due to the requirement that polymers gain viscoelastic behavior to better fit in the valleys of copper solid substrate, their $C A$ measurements were performed at high temperatures. Such measurements were carried out using a KRÜSS goniometer - Drop Shape Analyzer (DSA100) based on the sessile drop method (ADVANCE software). For the polymer films, $C A$ measurements were performed at high temperatures $\left(50\right.$ up to $\left.200^{\circ} \mathrm{C}\right)$ inside a temperature-controlled chamber (TC21) under a dry nitrogen environment. The chamber's insulation guaranteed a stable equilibrium related to heating rates during the measurements. They were carried out with 1-bromonaphthalene, 97\% (mostly dispersive) and ethylene carbonate, $98 \%$ (more polar than 1-bromonaphthalene), both from Sigma-Aldrich. Five measurements were performed at different spots of each substrate, and the results achieved presented considerable reproducibility regarding the mean and standard deviation.

For monolayer graphene on $\mathrm{Cu}$ foil, advancing and receding contact angle, respectively ( $C A_{\text {advancing }}$ and $C A_{\text {receding }}$ ) measurements were carried out at room temperature with deionized (DI) water (polar) and ethylene glycol (less polar than water). Such measurements allowed the evaluation of topographic (surface roughness) and chemical heterogeneities of the solid substrates since hysteresis $(\triangle C A)$ could be achieved by analyzing the difference between advancing and receding contact angles $(\triangle C A=$ $\left.C A_{\text {advancing }}-C A_{\text {receding }}\right)$. If the hysteresis $\triangle C A$ is a value less than $10^{\circ}$, then the surface evaluated can be considered as ideal with negligible chemical and topographic heterogeneities [25-28].

The SFE and its $\gamma_{\mathrm{p}}$ and $\gamma_{\mathrm{d}}$ components were obtained by using the harmonic mean equation [16] and applying the Fowkes model [29] aligned to the Owens, Wendt, Rabel and Kaelble (OWRK) model [17], which divides the SFE into polar and dispersive contribution. Note that, in the current paper, we did not have the intention to determine the validity of the model applied, but to estimate the SFE and its components to better understand the key factors controlling adhesion between materials during the DDT technique.

\subsection{Direct dry transfer of CVD graphene onto polymer films}

The DDT transfer was based on experiments performed by Fechine et al. [10]. Polymeric films were rinsed with isopropanol to clean the surface. CVD graphene was deposited on the $\mathrm{Cu}$ surface, and polymer films were submitted to $80^{\circ} \mathrm{C}$ for 10 minutes in order to eliminate water molecules and airborne contaminants from their surfaces $[20,30]$. Later on, $\mathrm{CVD}$ graphene on $\mathrm{Cu}$ and the polymer film were placed in contact in an uniaxial hydraulic press machine (UHPM), equipped with a heating system and pressure controller. After 2 minutes, the setup was removed from the equipment and the solid copper foil was manually removed using a tweezer leaving graphene on the polymer surface.

\subsection{Raman spectroscopy}

Raman spectra were carried out with a Witec Alpha 300R confocal Raman spectrometer. The excitation source was a $488 \mathrm{~nm}$ laser with a laser power below $10 \mathrm{~mW}$ for graphene film on the $\mathrm{Cu}$ foil and $\mathrm{a}$ 
$532 \mathrm{~nm}$ laser with a laser power below $2 \mathrm{~mW}$ on the graphene-polymer surface in order to avoid laser-induced local heating. Single spectra were carried out on different parts of the samples, and the data was analyzed using WITec Project software. The Raman Spectroscopy Mappings were performed at selected spots, chosen at random, with limitation across the region analyzed because of the graphene-polymer surface roughness due to the easy loss of focus at long lengths.

\subsection{Oscillatory rheology tests}

Rheological tests were performed in order to obtain the materials viscoelastic behavior in the melting flow by evaluating their storage modulus $\left(G^{\prime}\right)$, loss modulus $\left(G^{\prime \prime}\right)$, and complex viscosity $\left(\eta^{*}\right)$ as a function of the temperature. Temperature sweep tests were carried out in a Small Amplitude Oscillatory Shear (SAOS), from 120 to $220^{\circ} \mathrm{C}$, at $1 \mathrm{~Hz}$ frequency, $0.5 \%$ strain and $2{ }^{\circ} \mathrm{C} / \mathrm{min}$ linear heat ramp, with a suitable gap for each polymer. A Modular Compact Rheometer (MCR-102, Anton Paar, 25 mm diameter and plate-plate geometry) was used to execute these analyses.

\subsection{Molecular weight modification of polymers via dynamic thermomechanical degradation}

In order to modify the polymer's molecular weight, the dynamic thermomechanical degradation processes were performed in an Oscillatory Rheometer. Large Amplitude Oscillatory Shear (LAOS) tests were completed with constant shear strain $(100 \%)$ in the non-linear viscoelastic regime region at $120-200^{\circ} \mathrm{C}$,
$0.1 \mathrm{rad} / \mathrm{s}$ angular frequency, for 30 minutes under a compressed air environment.

\subsection{Fourier-transform infrared spectroscopy}

Polymer films were characterized using FourierTransform Infrared Spectroscopy (FTIR) via the Attenuated Total Reflectance (ATR) mode in order to compare the chemical structure of neat and modified polymers. The samples were allocated in a spectrometer, Cary 630 FTIR of AGILENT TECHNOLOGIES, in the range of 4000 to $650 \mathrm{~cm}^{-1}$.

\section{Results and discussion}

Graphene grown on $\mathrm{Cu}$ was characterized using Raman Spectroscopy. The representative Raman spectrum and mapping of the Full Width at Half Maximum (FWHM) of the 2D band of graphene are presented in Figure 1. The single spectrum exhibited in Figure 1a shows a fingerprint of graphene through a simple structure with the presence of two main bands designated as $\mathrm{G}$ and $2 \mathrm{D}$ bands of this material at 1589 and $2723 \mathrm{~cm}^{-1}$, respectively. In this case, a third band, the $\mathrm{D}$ band usually frequent on $1350 \mathrm{~cm}^{-1}$, did not appear in all spectra collected, indicating the absence or minimum presence of defects within the carbon lattice structure. The $\mathrm{G}$ band corresponds to the vibrational mode involving the $\mathrm{sp}^{2}$ hybridized carbon atoms comprising the graphene sheet, while the 2D band is a result of a two-phonon lattice vibrational process. The $\mathrm{D}$ band is known as a disorder band, representing edges or defects in the graphene structure. Here, the 2D band is very sharp, and it allowed the number of graphene layers to be determined through analyzing the $I_{2 \mathrm{D}} / I_{\mathrm{G}}$ ratio of

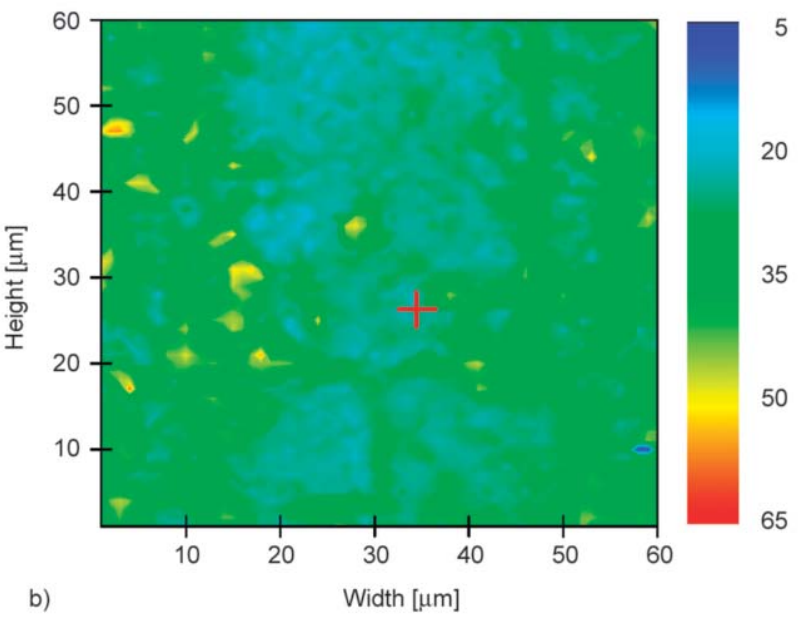

Figure 1. (a) Representative single spectrum and (b) Raman Mapping of Full Width at Half Maximum (FWHM) of a 2D band of CVD graphene grown on a copper substrate. 
these bands equal to 3.1 , classifying a high quality single-layer graphene. These factors, such as the ratio together with the lack of a D band and the strong presence of a 2D band are often used to confirm the quality defect free graphene substrate. Figure $1 \mathrm{~b}$ entails the FWHM Raman mapping of the 2D band in a specific area, also confirming the presence of single-layer graphene in almost the whole analyzed area since the values are between $25-30 \mathrm{~cm}^{-1}$ [31].

The contact angle results measured for graphene on the $\mathrm{Cu}$ substrate at room temperature corresponded to $87.5 \pm 2.1$ and $82.4 \pm 0.7^{\circ}$, for DI water $C A_{\text {advancing }}$ and $C A_{\text {receding, }}$, respectively, with a hysteresis value of $5^{\circ}$. On the other hand, the ethylene glycol $C A_{\text {advancing }}$ achieved was $74.9 \pm 0.9^{\circ}$ and $C A_{\text {receding }}$ was $70.5 \pm 2.5^{\circ}$ with a hysteresis value of $4^{\circ}$. The results indicated that metallic roughness and chemical heterogeneities are negligible, as the hysteresis $(\triangle C A)$ was less than $10^{\circ}$. In terms of the DI water contact angle (WCA), it is expected that ideal graphene presents values near $100-127^{\circ}$ due to its hydrophobicity [1], neglecting the influence of nature of the substrates. However, some authors suggested that such values can vary according the substrate on which graphene is deposited [4, 19, 32]. For instance, it was shown that $W C A$ measurements for the $\mathrm{Cu}$ substrate with and without a monolayer graphene coating seemed to be 86 and $84^{\circ}$, respectively, due to the wetting transparency effect [4]. The results presented here show a value of advancing $W C A 87^{\circ}$ approximately, which indicates strong influence of the $\mathrm{Cu}$ surface in such measurement.

SFE, polar, and dispersive components were calculated based on $C A_{\text {advancing }}$ measurements and are presented in Table 1. Besides, the adhesion between the 2D material and polymers could be inferred through

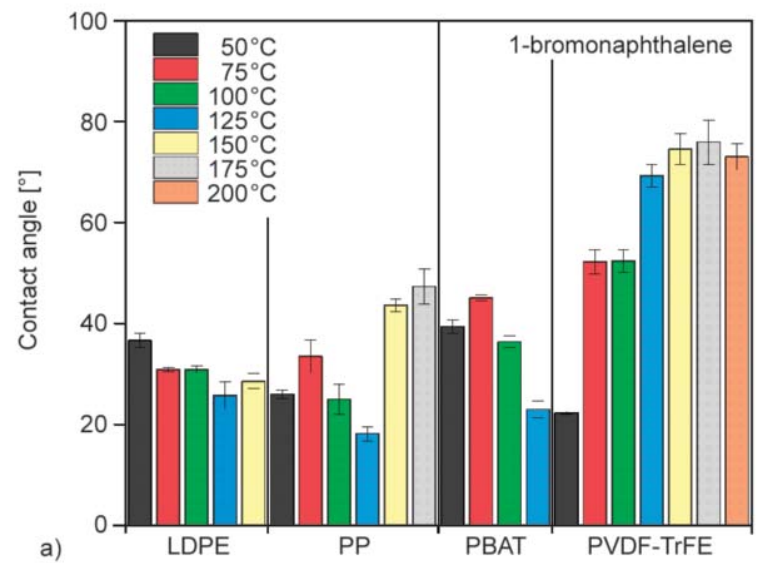

Table 1. Surface properties calculated for CVD graphene on the $\mathrm{Cu}$ substrate.

\begin{tabular}{|c|lll|r|}
\hline \multirow{2}{*}{$\begin{array}{c}\text { Surface } \\
\text { properties }\end{array}$} & Polar component & $\gamma_{\mathrm{p}}$ & {$\left[\mathrm{mJ} / \mathrm{m}^{2}\right]$} & $20.0 \pm 2.5$ \\
\cline { 2 - 5 } & Dispersive component & $\gamma_{\mathrm{d}}$ & {$\left[\mathrm{mJ} / \mathrm{m}^{2}\right]$} & $5.8 \pm 1.2$ \\
\cline { 2 - 5 } & Surface Free Energy & $S F E$ & {$\left[\mathrm{~mJ} / \mathrm{m}^{2}\right]$} & $25.8 \pm 2.2$ \\
\hline
\end{tabular}

the comparison of $\gamma_{\mathrm{p}}$ and $\gamma_{\mathrm{d}}$ values achieved for each material. For CVD graphene, measurements performed at room temperature mimicked the suitable transfer conditions to polymer surfaces because even at high temperatures, usually applied during DDT process, significant $S F E$ modifications were not expected due to the copper substrate maintaining its solid state even at temperatures above $200^{\circ} \mathrm{C}$, thus the graphene structure is also stable.

The polar component obtained of $20 \pm 2.5 \mathrm{~mJ} / \mathrm{m}^{2}$ is much more significant than the dispersive component that corresponded to $5.8 \pm 1.2 \mathrm{~mJ} / \mathrm{m}^{2}$ indicating the influence of the bond polarity character and hydrophilicity attributed to copper surface. Usually, an adhesion surface study of polymers and solid surfaces is performed based on $S F E$ values achieved at room temperature. However, such assumptions cannot be so reliable due to thermodynamic factors involving polymers; evidencing that $S F E$ tends to decrease as temperature rises owing to a great molecular movement with an insufficient package of polymeric chains [33, 34]. Here, all the data was collected at polymer temperature ranges above their glass transition temperature $\left(T_{\mathrm{g}}\right)$ values. Figure 2 shows the contact angle results as a function of temperature for the selected polymers.

The results indicated that LDPE and PP present a tendency to maintain stable contact angle values when ethylene carbonate was used even at high temperatures, where polymer chains acquired more

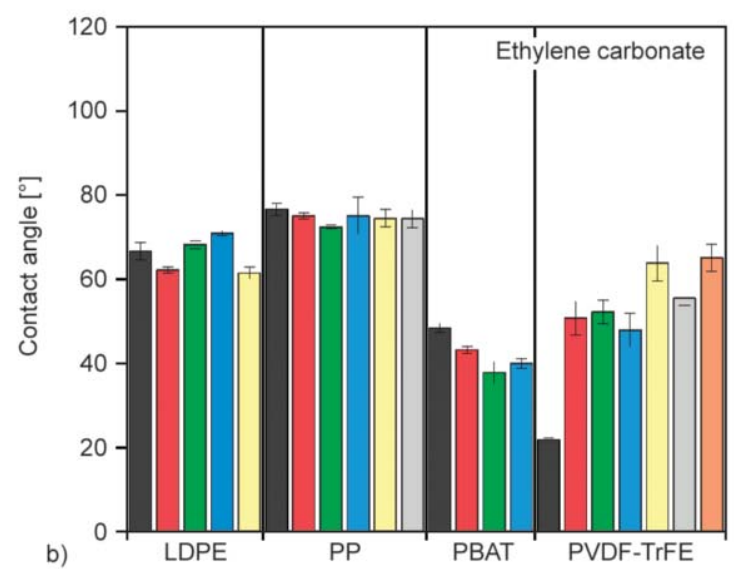

Figure 2. Contact angle measurements for all polymers at specific temperatures $\left(50-200^{\circ} \mathrm{C}\right)$ with (a) 1-bromonaphthalene and (b) ethylene carbonate. 
mobility. On the other hand, for 1-bromo-naphthalene $C A$ values the data is lower, which indicates that charge interactions between the polymer surface and the non-polar liquid are stronger than those with a polar liquid. The consequence of that is a wettability process more evident due to such polymer chain structure is predominantly non-polar and formed by carbon and hydrogen atoms. Measurements for LDPE films at $150^{\circ} \mathrm{C}$ could not be completed because at this temperature the surface was very heterogeneous containing topographic irregularities and air bubbles.

In the case of PBAT and PVDF-TrFE, a tendency for the $C A$ results to remain similar for both liquids was observed. PBAT only showed a significant modification at $125^{\circ} \mathrm{C}$ using 1-bromonaphthalene, probably because the chain mobility was greatly intensified due to the greater proximity to its melting temperature so non-polar interactions were favored. It may indicated that functional groups and mainly the aromatic rings presented on the polymer surface, when submitted to harsh thermal conditions, tend to be targeted in the viscous polymers mass providing superior wettability for non-polar liquid interaction. Above such temperature value, it was not possible to perform $C A$ measurements for PBAT due to surface irregularities related to melting process consequences. Nevertheless, $C A$ measures at temperatures closer to those applied during DDT technique $\left(125-130^{\circ} \mathrm{C}\right)$ was achieved, which made the calculation of polar and dispersive components feasible to infer the adhesion.

For PVDF-TrFE, a great increase at $75^{\circ} \mathrm{C}$ with 1bromoaphthalene was observed indicating that any thermal energy, even at distant temperatures below the melting point applied to the system, was enough to

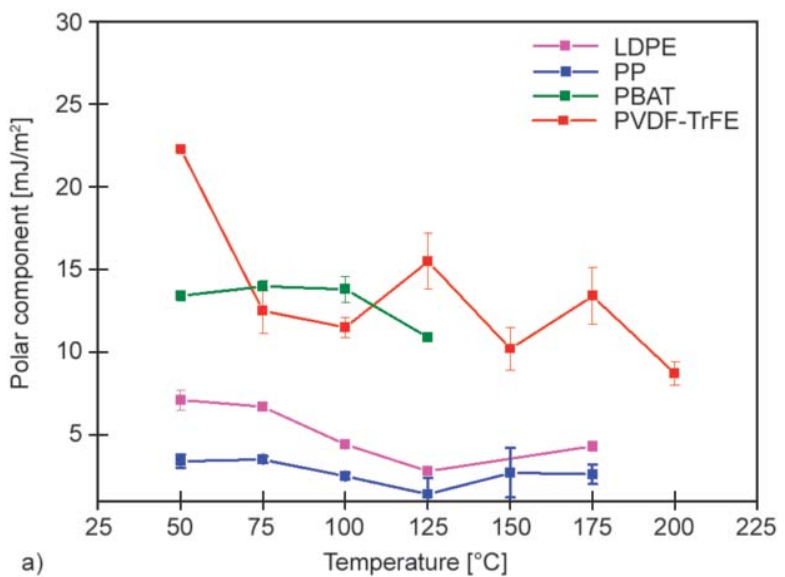

change the molecular arrangement of such polymer chains. Significant changes in the $C A$ results up to $125^{\circ} \mathrm{C}$ could not be observed, suggesting that the great mobility of polymer chains may be attributed to a higher disorder related to entropy in the system. Fluorine atoms composing the copolymer structure can be partially distributed and targeted inward of the polymer film and also to the surface, so very similar interactions occurred for both liquids. Although meaningful differences were not observed, a tendency to increase $C A$ values as a function of temperature in both cases was noted. It may be related to possible changes in solid-liquid interactions during spreading phenomena for each material, which include contacts between the three phases in this typical situation, a liquid wetting a solid in a gaseous environment.

This approach was attempted to further evaluate surface effects encompassing the adhesion behavior of CVD graphene on the polymer substrates. Accordingly, SFE was calculated as well as $\gamma_{\mathrm{p}}$ and $\gamma_{\mathrm{d}}$, for polymer films submitted to specific thermal conditions. Figure 3 shows $\gamma_{p}$ and $\gamma_{d}$ components values for each polymer in the temperature range used to measure the contact angle. Mostly, the results shown in Figure 3 indicated a possible effect of new spatial arrangements (conformation) of polymer molecular chains as the temperature increased, visibly generating variations in the polar and dispersive components. As the temperature range selected for each polymer was above its glass transition temperature $\left(T_{\mathrm{g}}\right)$, any increase in temperature was shown to be effective to promote modifications of the internal energy in the system.

Once again, thanks to the very similar chemical structure presented by LDPE and PP, such materials

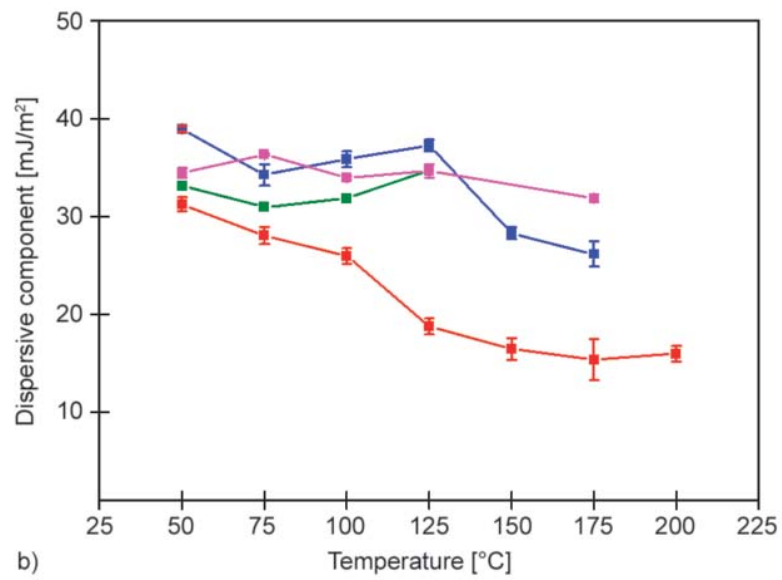

Figure 3. Surface analysis of selected polymers carried out at specific thermal conditions mimicking transfer process. (a) polar $\left(\gamma_{\mathrm{p}}\right)$ and $(\mathrm{b})$ dispersive $\left(\gamma_{\mathrm{d}}\right)$ components as a function of temperature $\left(50-200^{\circ} \mathrm{C}\right)$. 
exhibited similar behavior with lower values of polar components and higher values of dispersive components when compared to the copolymers. After increasing the temperature and, consequently, the thermal energy of the system, atoms and molecules located at the surface of polymers started to gain greater mobility to go through the whole material structure while there is a decrease in macroscopic stiffness, from which a peculiar effect of reconfiguration of the polymer chains was detected due to the molecules moving apart. Thereby, the average distance between individual molecular chains was increased as temperature increased followed by a reduction in polymer density and a rise in free volume. The PBAT presented values of polar and dispersive components that are more stable probably due to the restriction of polymer chain movement related to greater stiffness, even at high temperatures, essentially thanks to the aromatic hydrocarbon in the repeating unit of such structure. For PVDF-TrFE, the results indicated that the effect prompted by the temperature increase may have provide a rotation of fluorine atoms located on the polymer film surface, targeting it towards the bulk material, which may confirm the greater dispersive component value found.

Once polar and dispersive components for polymers (Figures 3a and 3b) and CVD graphene (Table 1) were determined, the adhesion capacity of both materials could be inferred. Table 2 shows the most suitable temperatures for polymers to be used as thermal condition in transfer process of CVD graphene. Such temperatures were selected considering the fittest thermal condition in which graphene and polymer showed greater imminence involving polar-polar and dispersive-dispersive values for these materials. The results indicated that for LDPE, PP, and PVDFTrFE, a temperature of $175^{\circ} \mathrm{C}$ would be the most appropriate, while, for PBAT a temperature of $125^{\circ} \mathrm{C}$ seemed to be the best choice.

Afterwards, since surface properties were studied in order to estimate the adhesion capacity between polymers and CVD graphene deposited on the solid substrates, the DDT process transfer method at such temperatures was also appraised. For this, tests involving the most appropriate temperatures mentioned previously for each polymer were completed (Table 2). This group of polymeric surfaces is identified here as neat polymers. Moreover, in order to evaluate the influence of molecular weight modification of polymers for graphene transfer processes, the polymers
Table 2. The most suitable temperatures selected according to a greater approximation between polar and dispersive components of CVD graphene and polymers to be used as thermal condition in graphene transfer process.

\begin{tabular}{|l|r|r|r|r|}
\hline \multicolumn{1}{|c|}{ Material } & $\begin{array}{c}\boldsymbol{T} \\
{\left[{ }^{\circ} \mathbf{C}\right]}\end{array}$ & $\begin{array}{c}\gamma_{\text {polar }} \\
{\left[\mathbf{m J} / \mathbf{m}^{2}\right]}\end{array}$ & $\begin{array}{c}\gamma_{\text {dispersive }} \\
{\left[\mathbf{m J} / \mathbf{m}^{2}\right]}\end{array}$ & $\begin{array}{c}\boldsymbol{S F E} \\
{\left[\mathbf{m J} / \mathbf{m}^{2}\right]}\end{array}$ \\
\hline CVD graphene & 20 & $20.0 \pm 2.5$ & $5.80 \pm 1.2$ & $25.8 \pm 2.2$ \\
\hline LDPE & 175 & $4.3 \pm 0.3$ & $31.9 \pm 0.4$ & $36.2 \pm 0.5$ \\
\hline PP & 175 & $2.6 \pm 0.6$ & $26.2 \pm 1.3$ & $28.8 \pm 1.4$ \\
\hline PBAT & 125 & $20.9 \pm 0.3$ & $34.8 \pm 0.4$ & $45.6 \pm 0.5$ \\
\hline PVDF-TrFE & 175 & $13.4 \pm 1.7$ & $15.4 \pm 2.1$ & $28.8 \pm 2.7$ \\
\hline
\end{tabular}

were submitted to dynamic thermomechanical degradation. CVD graphene was also transferred to these modified polymer samples by DDT technique using the same temperatures defined previously. Figure 4 shows maps of Full Width at Half Maximum (FWHM) of the 2D band associated with the graphene transferred to neat and modified polymer surfaces, with the respective percentage of graphene coverage reached at the temperature tests mentioned.

Initially, it was possible to transfer $75 \%$ of CVD graphene to LDPE at $175^{\circ} \mathrm{C}$ (Figure 4a). However, no quantity could be transferred to $\mathrm{PP}$ at $175^{\circ} \mathrm{C}$, in which no CVD graphene signal was detected. These results are an indication that analyzing only surface properties may be not enough to define transfer results and adhesion between the polymer and graphene, because other factors can also influence this process, such as the chemical structure and rheological properties [10]. For PBAT, at $125^{\circ} \mathrm{C}$, it was possible to transfer $68 \%$ of CVD graphene (Figure $4 \mathrm{~b})$. On the other hand, it was possible to cover only $8 \%$ of PVDF-TrFE at $175^{\circ} \mathrm{C}$ (Figure $4 \mathrm{c}$ ). In general, LDPE and PBAT seemed to be more favorable to adhesion with the 2D material evaluated (Figures $4 \mathrm{a}$ and $4 \mathrm{~b}$ ). For modified polymer surfaces, an improvement in the percentage of transferred graphene for all cases in comparison with the same type of neat material was noticed. For LDPE, $90 \%$ was acquired (Figure 4d), for PBAT 85\% (Figure 4e) and for PVDFTrFE it was possible to achieve $40 \%$ of $2 \mathrm{D}$ material coating (Figure 4f). Except for PP, in which, again, it was not possible to transfer any quantity of the 2D material due to non-favorable chemical structure aligned to the surface properties, indicating low tack.

In the interest of evaluating the influence of the rheological properties of polymers, oscillatory rheological tests were performed on neat and modified polymers. Figure 5 presents rheological curves achieved for 

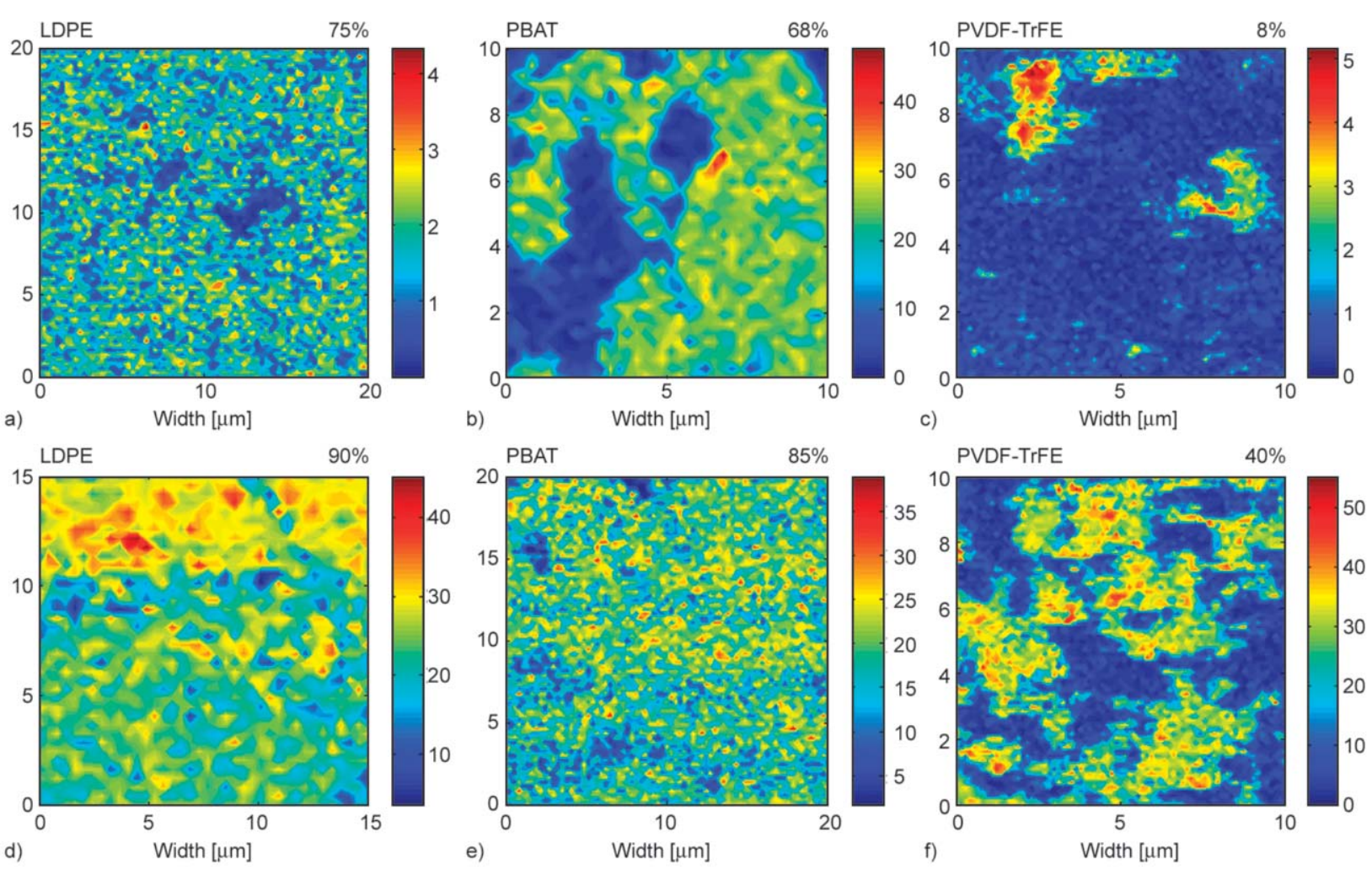

Figure 4. Raman characterization results for neat and modified polymers. FWHM 2D mapping and the percentage of single-layer graphene coating for neat (a) LDPE, (b) PBAT, (c) PVDF-TrFE and modified polymers (d) LDPE, (e) PBAT and (f) PVDF-TrFE.

each condition. The results obtained in Figures 5a and $5 \mathrm{~b}$ shows that both neat and modified polymer exhibited storage modules $\left(G^{\prime}\right)$ that did not exceed $10^{5} \mathrm{~Pa}$, corroborating to Dahlquist criterion [35], which is based on molecular motions and determines the adhesion between a viscoelastic material and solid surface, such as metals. This indicates that polymeric materials have the capability to deform and promote satisfactory contact with the solid substrate, which means that the molten polymer denotes a proper viscosity so that the surface regions of the valleys of the solid substrate can be filled. Moreover, the loss modulus $\left(G^{\prime \prime}\right)$ acquired for neat and modified polymers, respectively, presented a minimum value showing that such materials exhibited a viscous behavior low enough to be able to dissipate the necessary energy during the solidification step. At the same time, for both it was not possible to verify significant modifications. This condition leads to a polymer/ graphene adhesion greater than the copper/graphene binding energy, so consequently, the graphene can be easily transferred to polymer surface [10].

Correlating these two rheological characteristics $\left(G^{\prime}\right.$ and $\left.G^{\prime \prime}\right)$, the complex viscosity ( $\left.\eta^{*}\right)$ could be calculated. In accordance with Fechine et al. [10], there is an optimal value in the range of $8000 \mathrm{~Pa} \cdot \mathrm{s}$ in which neat polymers tend to promote better tack to solid substrates. Figures $5 \mathrm{c}$ and $5 \mathrm{~d}$ shows the complex viscosity results achieved for neat and modified polymers, respectively, which corroborate the transfer results presented previously, with higher percentage values of CVD graphene coating on the LDPE (75\%) (Figure 4a) and PBAT (68\%) (Figure 4b) surfaces because such polymers presented the most favorable $\eta^{*}$, nearly to $8000 \mathrm{~Pa} \cdot \mathrm{s}$. By contrast, neat PP and neat PVDF-TrFE presented values of $\eta^{*}$ much more distinct, justifying the very low percentage of coating of PVDF-TrFE (8\%) (Figure 4c) with CVD graphene while for the PP surface, no quantity of such 2D material could be transferred.

Concerning complex viscosity, a notable decrease in the values of PVDF-TrFE and PP were observed for modified polymers (Figure 5d), registering a modification of molecular weight due to dynamic thermomechanical degradation, altering, therefore, the complex viscosity of such polymers. Modified LDPE (Figure 5d) showed very similar viscoelastic behavior of those exhibited to the same neat polymer (Figure 5c), indicating that thermomechanical degradation was not enough to modify its rheological 

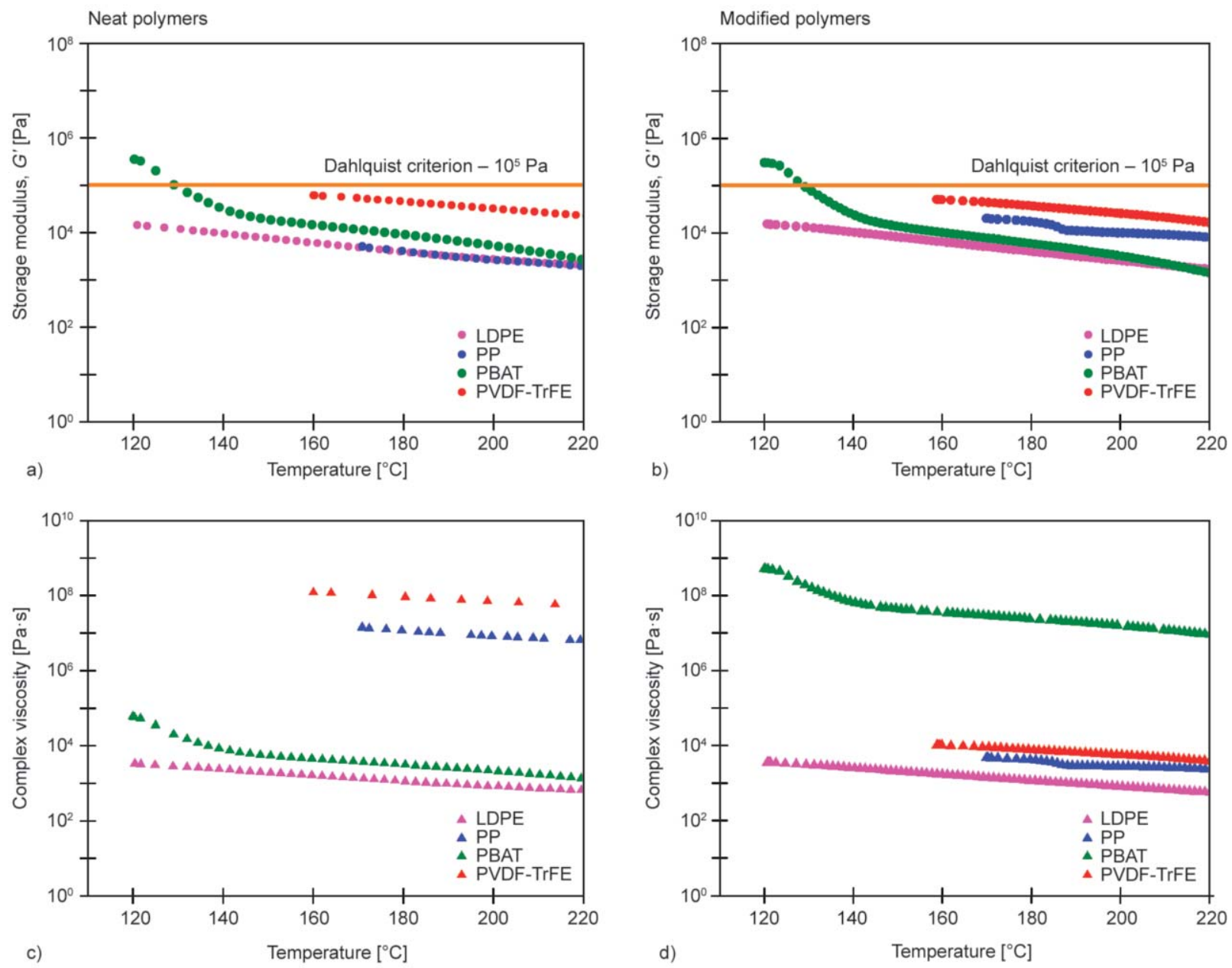

Figure 5. Rheological curves achieved for neat and modified polymers, as a function of temperature: (a) and (b) storage modulus $\left(G^{\prime}\right)$, (c) and (d) complex viscosity $\left(\eta^{*}\right)$, respectively.

properties; however, an increase in the graphene quantity transferred to the polymer surface was noted (Figure 4a). Modified PP (Figure 5d) showed a diminution of complex viscosity; however, the value reached was not sufficient to guarantee any transfer percentage, indicating that such polymer is not presented as an ideal candidate for successful graphene transfer process. Modified PBAT (Figure 5d) presented higher complex viscosity than the same neat polymer (Figur $5 \mathrm{c}$ ), suggesting that reticulation reaction processes may be have occurred, resulting in a greater resistance to flow. Here, the value of complex viscosity in the range of $8000 \mathrm{~Pa} \cdot \mathrm{s}$ seemed to be a minimum value correlated to an ideal value based on literature which is associated with molecular weight and the chemical structure of polymers. However, the increase in the complex viscosity to $7 \cdot 10^{8} \mathrm{~Pa} \cdot \mathrm{s}$ (Figure 5d) allowed up to $85 \%$ of graphene coverage on this polymer surface (Figure 4e), an improvement higher than $15 \%$ clearly observed in Figure $4 b$, when compared to the adhesion process as the Dahlquist criterion is maintained for neat and modified PBAT (Figures 5a and 5b).

Initially, rheological results indicated that it would not be possible to transfer CVD graphene onto neat PVDF-TrFE surface (Figure 5c), since its complex viscosity was not near to $8000 \mathrm{~Pa} \cdot \mathrm{s}$, and very low percentages of coatings were achieved in the order of only $8 \%$ (Figure $4 \mathrm{c}$ ), probably because of its nonfavorable chemical structure configuration during adhesion process. However, after thermomechanical degradation, a reduction in complex viscosity was observed (Figure 5d) indicating cleavage of polymer chains and because of that an increase in the percentage of CVD graphene transferred (40\%) to PVDFTrFE was observed (Figure 4f). It was concluded that by the insertion of thermal energy and mechanical deformation in the polymer chain arrangement, a greater adhesion between molten polymers and the copper surface could be attained. It is suggested that the results are connected to the thermal degradation that may have generated oxidation of such polymeric 
structures as a result of elevated temperatures applied to the systems. On the other hand, the mechanical deformation may have broken polymer chains due to a physical force, such as strain force. The complex viscosity of PBAT increased in comparison to PVDF-TrFE and PP, resulting in an improvement in coverage of the transferred graphene. On the other hand, even if the complex viscosity of PVDF-TrFE had decreased, the graphene coverage was also improved.

In a brief, the results presented allowed the evaluation of the viscoelastic behavior of polymers, which includes their viscosities. The ability of a material to flow is related to this rheological property and, in this case, the length of molecular chains was correlated to their molecular weight seeing that smaller chains present major capacity to flow easily while for the bigger ones, it is more difficult. It means that the molecular weight of these materials was inferred and, since modifications in their complex viscosity were observed, such analysis indicated that modified polymers are better candidates as target substrates in graphene transfer processes in comparison to neat polymers. Moreover, according to the literature, the crosslinking of polymer chains eventually increase their molecular weight [36]. Here, for instance, this behavior was clearly observed for PBAT (Figure $5 \mathrm{c}$ and $5 d$ ), in which higher values of complex viscosity were achieved and since reticulation processes occurred, its molecular weight was improved. Clearly, a specific behavior for each material was observed, which means that reticulated PBAT saw an improvement in the adhesion process with graphene. Here, it is of paramount importance to mention that for modified polymers, the irregular polymer surfaces did not allow contact angle measurements to be performed as the dynamic thermomechanical degradation have produced deterioration in the polymer chains structures.

Moreover, chemical modifications may induce the reduction of the complex viscosity of the modified polymers, which led to an enhancement in the process that can be observed in FTIR results in Figure 6. Such measurements performed in ATR mode allowed the analysis in a range of $2-3 \mu \mathrm{m}$ of the thickness of the polymer films, so the effect of chemical structure modification had occurred during the dynamic thermomechanical degradation, which was performed to modify the molecular weight of polymers was evaluated.
FTIR spectra indicated that the thermomechanical degradation did not significantly alter the chemical structure of the polymers, except for PVDF-TrFE and LDPE as can be observed in Figure 6. Polymers as PP (Figure 6b) and PBAT (Figure 6c) presented characteristic spectra referent to these type of materials and did not show alterations after the degradation process, which suggests that their surface chemical structure did not suffer great changes. For PP, the results involving chemical structure corroborates to surface properties analysis (Table 2) indicating that this polymer showed little possibility of adhesion owing to differences between polar and dispersive components in comparison to CVD graphene results. In contrast, LDPE (Figure 6a) and PVDF-TrFE (Figure 6d) presented modifications of the chemical structures due to thermal degradation. This was observed because of the presence of bands in the region of 1248 and $1157 \mathrm{~cm}^{-1}$ for LDPE sample, corresponding to $\mathrm{C}-\mathrm{O}$ band and symmetric stretching vibration of $\mathrm{C}-\mathrm{O}-\mathrm{C}$ band [37]. Meanwhile, for PVDF-TrFE an additional band at $1730 \mathrm{~cm}^{-1}$ corresponding to carbonyl stretch $(\mathrm{C}=\mathrm{O})$ was noted. Thus, the improvement of the percentage of CVD graphene coating may also be associated to chemical structure modification together with changes in rheological properties.

\section{Conclusions}

In summary, the surface properties of four kinds of polymers (LDPE, PP, PBAT and PVDF-TrFE) and of CVD graphene grown on a copper substrate have been studied in order to be used as a key to understanding the adhesion process between these materials. The understanding of this phenomenon could improve the quality of graphene transfer process and the performance of the devices built using this technique. Since the DDT transfer process is carried out at temperatures close to $T_{\mathrm{m}}$ of polymers, here, for the first time, contact angle measurements at high temperatures were achieved for the polymers. Using this data, a simple way to pre-estimate the efficiency of CVD graphene transfer method was presented, owing to an understanding of polymers surface properties. The current approach can be applied to any other 2D material transfer method besides Direct Dry Transfer (DDT), in order to obtain more data to rearrange transfer parameters or even to provide previous knowledge of what are the most suitable polymeric substrates for graphene transfers, regarding adhesion. 

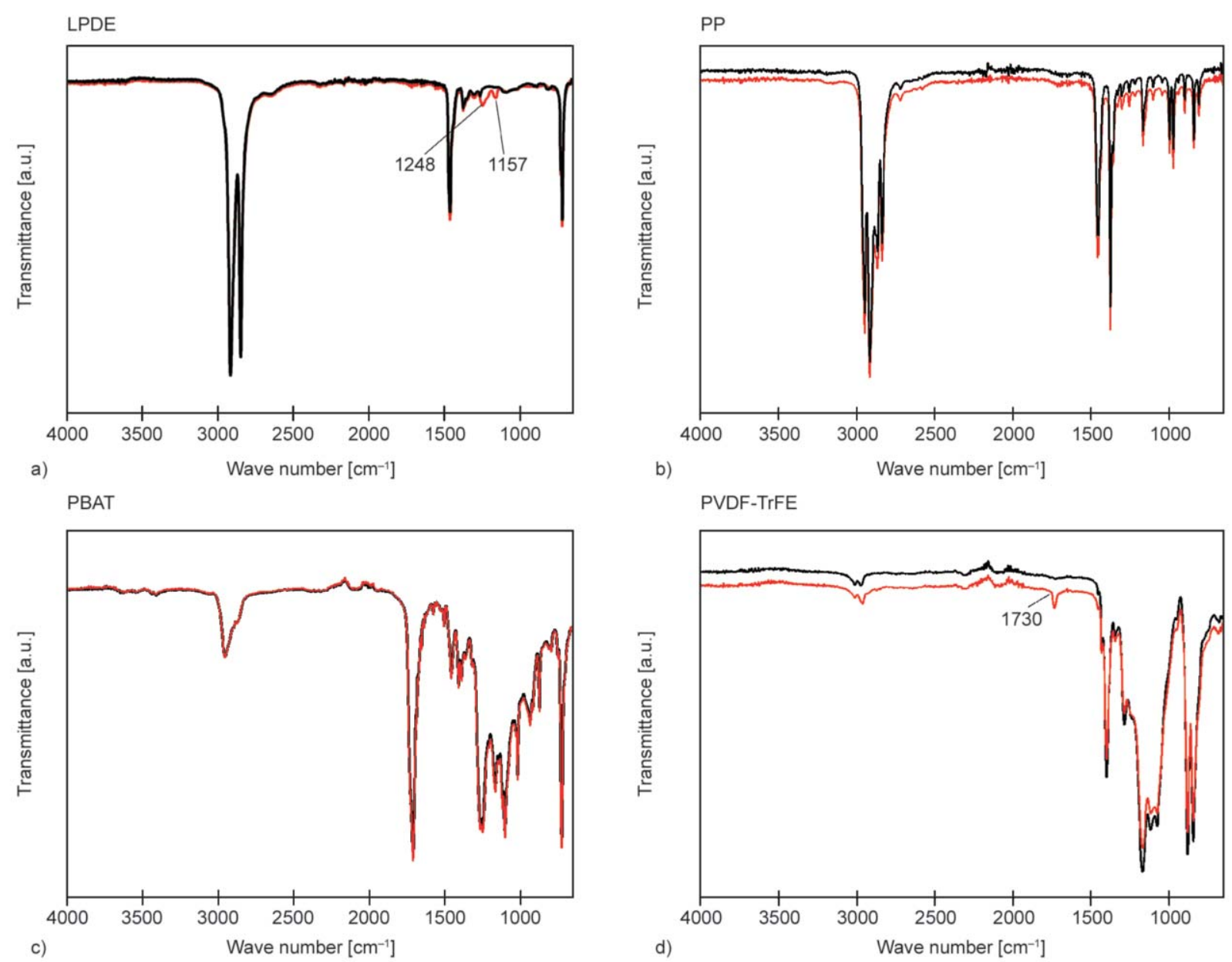

Figure 6. FTIR spectra of neat (black line) and modified (red line) polymers used in DDT processes (a) LDPE (b) PP, (c) PBAT and (d) PVDF-TrFE.

The adhesion capacity of the materials was inferred by analyzing their polar and dispersive components, and the best temperature for the transfer process could be determined. However, the surface properties seemed to not only be the necessary parameter to be evaluated in the adhesion between molten polymers and graphene, so rheological properties and chemical structures of polymers were also evaluated concomitantly. It has been shown that rheological properties can vary according to the molecular weight of polymers and it can influence the adhesion involving polymers and graphene, consequently, the quality and efficiency of the graphene transfer to such substrates. The results presented here suggests that polymer modification, such as molecular weight alteration and additional groups formations, induced a better performance of the graphene transfer process and allowed a thorough understanding of the role of different factors in adhesion phenomena and how they can be used to improve the percentage of graphene coating.

\section{Acknowledgements}

We acknowledge funding from the São Paulo Research Foundation (FAPESP), grants 2012/50259-8 and 2014/22840-3. This work is also partially funded by Fundo Mackenzie de Pesquisa (MackPesquisa). M.R.M.S. was supported by an undergraduate fellowship from FAPESP (2016/09608-0) and M.C.F.C. also acknowledges the Master Degree fellowship from CAPES.

\section{References}

[1] Wang S., Zhang Y., Abidi N., Cabrales L.: Wettability and surface free energy of graphene films. Langmuir, 25, 11078-11081 (2009).

https://doi.org/10.1021/la901402f

[2] Annamalai M., Gopinadhan K., Han S. A., Saha S., Park H. J., Cho E. B., Kumar B., Patra A., Kim S-W., Venkatesan T.: Surface energy and wettability of van der Waals structures. Nanoscale, 8, 5764-5770 (2016). https://doi.org/10.1039/C5NR06705G

[3] Bunch J. S., Verbridge S. S., Alden J. S., van der Zande A. M., Parpia J. M., Craighead H. G., McEuen P. L.: Impermeable atomic membranes from graphene sheets. Nano Letters, 8, 2458-2462 (2008). https://doi.org/10.1021/n1801457b 
[4] Rafiee J., Mi X., Gullapalli H., Thomas A. V., Yavari F., Shi Y., Ajayan P. M., Koratkar N. A.: Wetting transparency of graphene. Nature Materials, 11, 217-222 (2012).

https://doi.org/10.1038/nmat3228

[5] Berman D., Erdemir A., Sumant A. V.: Graphene: A new emerging lubricant. Materials Today, 17, 31-42 (2014).

https://doi.org/10.1016/j.mattod.2013.12.003

[6] Johnson W. C., Wang J., Chen Z.: Surface structures and properties of polystyrene/poly(methyl methacrylate) blends and copolymers. Journal of Physical Chemistry B, 109, 6280-6286 (2005).

https://doi.org/10.1021/jp045647a

[7] Ton-That C., Shard A. G., Daley R., Bradley R. H.: Effects of annealing on the surface composition and morphology of PS/PMMA blend. Macromolecules, 33, 8453-8459 (2000).

https://doi.org/10.1021/ma000792h

[8] Chen X., Zhang L., Chen S.: Large area CVD growth of graphene. Synthetic Metals, 210, 95-108 (2015). https://doi.org/10.1016/j.synthmet.2015.07.005

[9] Kang J., Shin D., Bae S., Hong B. H.: Graphene transfer: Key for applications. Nanoscale, 4, 5527-5537 (2012). https://doi.org/10.1039/C2NR31317K

[10] Fechine G. J. M., Martin-Fernandez I., Yiapanis G., Bentini R., Kulkarni E. S., Bof de Oliveira R. V., Hu X. Yarovsky I., Neto A. H. C., Özyilmaz B.: Direct dry transfer of chemical vapor deposition graphene to polymeric substrates. Carbon, 83, 224-231 (2015).

https://doi.org/10.1016/j.carbon.2014.11.038

[11] Kessler F., da Rocha C. O., Medeiros G. S., Fechine G. J. M.: Chemical vapor deposition graphene transfer process to a polymeric substrate assisted by a spin coater. Materials Research Express, 3, 035601/1-035601/9 (2016).

https://doi.org/10.1088/2053-1591/3/3/035601

[12] Silverstein T. P.: Polarity, miscibility, and surface tension of liquids. Journal of Chemical Education, 70, 253 (1993). https://doi.org/10.1021/ed070p253

[13] Finn K. H.: The measurement of surface energy of polymers by means of contact angles of liquids on solid surfaces - A short overview of frequently used methods. Department of Chemistry, Oslo (2004).

[14] Gent A. N., Hamed G. R.: Fundamentals of adhesion. in 'Handbook of adhesives' (ed.: Skeist I.) Springer, Boston, 39-73 (1990).

[15] Wu S.: Calculation of interfacial tension in polymer systems. Journal of Polymer Science Part C: Polymer Symposia, 34, 19-30 (2007). https://doi.org/10.1002/polc.5070340105

[16] Shimizu R. N., Demarquette N. R.: Evaluation of surface energy of solid polymers using different models. Journal of Applied Polymer Science, 76, 1831-1845 (2000).

https://doi.org/10.1002/(SICI)10974628(20000620)76:12<1831::AID-APP14>3.0.CO;2-Q
[17] Owens D. K., Wendt R. C.: Estimation of the surface free energy of polymers. Journal of Applied Polymer Science, 13, 1741-1747 (1969). https://doi.org/10.1002/app.1969.070130815

[18] Kaeble D. H.: Dispersion-polar surface tension properties of organic solids. The Journal of Adhesion, 2, 6681 (1972). https://doi.org/10.1080/0021846708544582

[19] Raj R., Maroo S. C., Wang E. N.: Wettability of graphene. Nano Letters, 13, 1509-1515 (2013). https://doi.org/10.1021/nl304647t

[20] Aria A. I., Kidambi P. R., Weatherup R. S., Xiao L., Williams J. A., Hofmann S.: Time evolution of the wettability of supported graphene under ambient air exposure. The Journal of Physical Chemistry. C, 120, 22152224 (2016). https://doi.org/10.1021/acs.jpcc.5b10492

[21] van Engers C. D., Cousens N. E. A., Babenko V., Britton J., Zappone B., Grobert N., Perkin S.: Direct measurement of the surface energy of graphene. Nano Letters, 17, 3815-3821 (2017).

https://doi.org/10.1021/acs.nanolett.7b01181

[22] Shin Y. J., Wang Y., Huang H., Kalon G., Wee A. T. S., Shen Z., Bhatia C. S., Yang H.: Surface-energy engineering of graphene. Langmuir, 26, 3798-3802 (2010). https://doi.org/10.1021/la100231u

[23] Zosel A.: Adhesion and tack of polymers: Influence of mechanical properties and surface tensions. Colloid and Polymer Science, 263, 541-553 (1985).

https://doi.org/10.1007/BF01421887

[24] Kessler F., Muñoz P. A. R., Phelan C., Romani E. C., Larrudé D. R. G., Júnior F. L. F., de Souza E. A. T., de Matos C. J. S., Fechine G. J. M.: Direct dry transfer of CVD graphene to an optical substrate by in situ photopolymerization. Applied Surface Science, 440, 55-60 (2018).

https://doi.org/10.1016/j.apsusc.2018.01.142

[25] Butt H. J., Graf K., Kappl M.: Physics and chemistry of interfaces. Wiley, Heppenheim (2003).

[26] Adamson W. A.: Physical chemistry of surfaces. Wiley, New York (1982).

[27] Choi S. W., Lauer H., Wenz G., Burns M., Petri D. F. S.: Formation of dense cellulose monolayers on silver surfaces. Journal of the Brazilian Chemical Society, 11, $11-15$ (2000). https://doi.org/10.1590/S0103-50532000000100003

[28] Damato T. C., Carrasco L. D. M., Carmona-Ribeiro A. M., Luiz R. V., Godoy R., Petri D. F. S.: The interactions between surfactants and the epicuticular wax on soybean or weed leaves: Maximal crop protection with minimal wax solubilization. Crop Protection, 91, 5765 (2017).

https://doi.org/10.1016/j.cropro.2016.09.019

[29] Fowkes F. M.: Attractive forces at interfaces. Industrial and Engineering Chemistry, 56, 40-52 (1964). https://doi.org/10.1021/ie50660a008 
[30] Li Z., Wang Y., Kozbial A., Shenoy G., Zhou F., McGinley R., Ireland P., Morganstein B., Kunkel A., Surwade S. P., Li L., Liu H.: Effect of airborne contaminants on the wettability of supported graphene and graphite. Nature Materials, 12, 925-931 (2013). https://doi.org/10.1038/nmat3709

[31] Malard L. M., Pimenta M. A., Dresselhaus G., Dresselhaus M. S.: Raman spectroscopy in graphene. Physics Reports, 473, 51-87 (2009).

https://doi.org/10.1016/j.physrep.2009.02.003

[32] Shih C-J., Wang Q. H., Lin S., Park K-C., Jin Z., Strano M. S., Blankschtein D.: Breakdown in the wetting transparency of graphene. Physical Review Letters, 109, 176101/1-176101/5 (2012).

https://doi.org/10.1103/PhysRevLett.109.176101

[33] Wang G., Gao E., Dai Z., Liu L., Xu Z., Zhang Z.: Degradation and recovery of graphene/polymer interfaces under cyclic mechanical loading. Composites Science and Technology, 149, 220-227 (2017).

https://doi.org/10.1016/j.compscitech.2017.06.004
[34] Zhao Q., Liu Y., Abel E. W.: Effect of temperature on the surface free energy of amorphous carbon films. Journal of Colloid and Interface Science, 280, 174-183 (2004). https://doi.org/10.1016/j.jcis.2004.07.004

[35] Heddleson S. S., Hamann D. D., Lineback D. R.: The Dahlquist criterion: Applicability of a rheological criterion to the loss of pressure-sensitive tack in flourwater dough. Cereal Chemistry, 70, 744-748 (1993).

[36] Yoshida S., Hagiwara K., Hasebe T., Hotta A.: Surface modification of polymers by plasma treatments for the enhancement of biocompatibility and controlled drug release. Surface and Coatings Technology, 233, 99-107 (2013).

https://doi.org/10.1016/j.surfcoat.2013.02.042

[37] Zarandi M. B., Bioki H. A.: Thermal and mechanical properties of blends of LDPE and EVA crosslinked by electron beam radiation. The European Physical Journal Applied Physics, 63, 21101/1-21101/7 (2013).

https://doi.org//10.1051/epjap/2013130238 